\title{
Misoprostol como abortivo en España. A propósito de un caso de autopsia judicial.
}

\author{
Misoprostol as an abortive in Spain. Report of a judicial \\ autopsy case.
}

\section{PM. Garamendi González¹ y MI. Landa Tabuyo ${ }^{1}$}

\section{RESUMEN}

El Misoprostol es un agente farmacológico introducido en el mercado español bajo la forma de un fármaco protector gástrico y comercializado actualmente en asociación con un antinflamatorio. Desde hace varios años, se desarrolla un debate internacional sobre el hecho de que este mismo producto cumple las características de un abortivo extremadamente eficaz. No obstante, el uso inadecuado del Misoprostol puede causar daños severos a la madre $y$ al feto, tanto si el producto se usa con fines abortivos como si se administra accidentalmente durante una gestación, incluso a las dosis recomendadas. Por otra parte, el Misoprostol tiene un rápido metabolismo y no es fácilmente identificable por los laboratorios toxicológicos de referencia, limitando su posible identificación en casos de uso inadecuado, voluntario $o$ accidental. Se presenta un caso de autopsia médico legal tras un posible episodio de aborto inducido por la administración de Misoprostol.

Palabras clave: Aborto, cuestiones éticas, identificación toxicológica, malformación fetal, Misoprostol.

\section{ABSTRACT}

Misoprostol is a drug commercialized in Spain as a gastric protector. Nowadays it is sold in our country also associated with diclofenac to prevent gastric damages produced by anti-inflammatories. During the last few years, there has been a public debate in medical press about the fact that Misoprostol has also proved to be an excellent abortive. Nevertheless, when it is not properly used Misoprostol can be the cause of severe damages to the fetus and the mother. This Prostaglandin EI analog is a drug rapidly metabolized in humans and it is not easy to identify in toxicological analyses. This is the main bias when trying to identify it in forensic cases of accidental damages after using it as a gastric protector or voluntary administration as an abortive. We present a case report about a forensic autopsy following a possible Misoprostol induced abortion.

Key words: Abortion, ethics, fetal malformation, Misoprostol, toxicological identification.

Este trabajo no ha contado con financiación por parte de ningún organismo público o privado.

Correspondencia: Pedro M. Garamendi. Servicio de Clínica Médico Forense. Subdirección de Vizcaya. Instituto Vasco de Medicina Legal. C/ Buenos Aires nº 6. Planta 4a 48001 Bilbao. E-mail: garamendi.pm@aju.ej-gv.es.

${ }^{1}$ Médico Forense. Especialista en Medicina Legal y Forense. 


\section{INTRODUCCIÓN:}

Se estima que cada año se realizan en el mundo 19 millones de abortos de riesgo fuera del entorno sanitario y como consecuencia de ellos sucede la muerte de un total de 70.000 mujeres cada año. El número de malformaciones y anomalías fetales producidas por el uso de agentes teratogénicos de forma incontrolada con fines abortivos resulta incalculable [I]. En muchos países todas las formas de aborto son ilegales, con la excepción de los casos de embarazos tras violaciones o en casos de riesgos para la salud de la madre. Esta circunstancia ha condicionado en estos países una proliferación de los abortos ilegales, en muchos casos fuera del contexto sanitario y sin la adecuada supervisión médica aplicando métodos en ocasiones inapropiados. Brasil, donde el aborto más allá de los supuestos indicados es ilegal, fue uno de los primeros países en identificar el uso generalizado con fines abortivos fuera del contexto sanitario de una sustancia originalmente descrita en la terapéutica como protector gástrico: el Misoprostol [2].

El Misoprostol es un producto extremadamente útil como abortivo. Tiene un alto margen de seguridad cuando es pautado en dosis adecuadas y con la adecuada supervisión médica y es muy eficaz como inductor de dilatación cervical y contracciones uterinas. Por otra parte, es un producto más barato que los abortivos oficialmente utilizados. Pero, también, el Misoprostol es un producto de metabolización y biodisponibilidad muy rápida y, al tiempo, difícilmente detectable por los laboratorios de referencia en toxicología forense: todo ello lo convierte en un abortivo ilegal ideal.

Pese a su elevado margen de seguridad cuando es utilizado bajo supervisión médica, las referencias sobre complicaciones para el feto y para la madre en casos de uso de esta sustancia como abortivo son múltiples en la bibliografía mundial. Estas complicaciones son más acusadas y severas en los casos de uso de esta sustancia en dosis inadecuadas y sin el correcto control sanitario. Por ello, además, es un abortivo relativamente seguro pero potencialmente peligroso en el caso de ser usado fuera del contexto sanitario.

En España el Código Penal considera circunstancias de exclusión de responsabilidad penal por la inducción de aborto (Disposición Derogatoria Primera de la Ley 10/1995, relativa a la no derogación del artículo 4I7 bis introducido por la Ley Orgánica 9// 985 en la redacción del Código Penal de 1973):

- Cuando el aborto sea necesario para evitar un grave peligro para la vida o la salud física o psíquica de la embarazada.

- Embarazos producto de delito previo de violación, cuando el aborto se practica en las primeras doce semanas de gestación.

- Cuando se presuma que el feto nacerá con graves taras físicas o psíquicas, cuando se practique en las veintidós primeras semanas de gestación.

Estas circunstancias exigen que el aborto provocado no punible sea realizado por un médico o bajo su dirección y constando consentimiento expreso de la mujer embarazada, salvo en el caso de urgencia por riesgo vital en los casos del primer supuesto [3].

Se presenta un caso de autopsia médico legal practicada tras un posible aborto inducido en el segundo trimestre de gestación fuera del entorno sanitario mediante el uso de Misoprostol en dosis inadecuadas. La presentación del caso abre una discusión sobre las características toxicológicas del Misoprostol, sus posibles interferencias con la investigación médico legal de los casos en los que su uso se haya producido y sobre las posibles implicaciones éticas de su uso con fines abortivos en nuestro país. 


\section{PRESENTACIÓN DEL CASO:}

La madrugada del día 25 al 26 de julio de 1998 se practica en el barrio de Cortes de la localidad de Bilbao el levantamiento del cadáver de un feto hallado en el interior de un contenedor de basuras. El feto se halla en el interior de una bolsa de plástico en la que se encuentran, además del feto, restos de placenta, cordón umbilical, un cabello y una compresa con restos de sangre.

Las pesquisas de la Ertzaintza (policía autónoma vasca) permiten localizar en pocas horas a una mujer que supuestamente habría sido la madre del feto. Según las primeras declaraciones de la supuesta madre, esta reconoce ante los agentes que había utilizado como inductor del aborto fuera del contexto médico una sustancia comercial denominada Cytotec ${ }^{\circledR}$. Las dosis utilizadas han sido de unos 20 comprimidos de esta sustancia por vía oral y otros 20 comprimidos por vía vaginal.

La tarde del día 26 de julio, la madre pasa a disposición judicial y es reconocida por el médico forense de guardia. Este realiza una exploración general en la que destaca el hecho de que la mujer refiere consumo habitual de cocaína por vía parenteral y metadona por vía oral. Se recoge muestra de orina de la madre para estudio toxicológico y sangre materna para estudio de maternidad.

La autopsia se practica en la mañana del día 26 de julio. Los hallazgos macroscópicos correspondieron con un feto varón de $30 \mathrm{~cm}$ de longitud vértex-talón, $14 \mathrm{~cm}$ occipito-sacro, $17,5 \mathrm{~cm}$ de perímetro torácico, $20 \mathrm{~cm}$ de perímetro craneal y $555 \mathrm{gr}$ de peso total. Al examen externo, se encuentra ausencia de lanugo o vérmix caseosa, hendiduras palpebrales cerradas, aparato genital inmaduro con cierre de procesos escrotales, orificio anal permeable, extremidades con definición completa. Los datos de evolución cadavérica indican la existencia de livideces declive mínimas, rigidez no valorable, piel húmeda con signos de desecación en extremidades y ausencia de mancha verde. No se apreciaron signos externos sugestivos de violencia traumática específica.

El examen interno indicó:

- encéfalo: 62 gr.

- corazón: 3,2 gr.

- pulmones: 10 y $8,6 \mathrm{gr}$.

- timo: 2,3 gr.

- tiroides: 0,4 gr.

- bazo: 0,4 gr.

- hígado: $29 \mathrm{gr}$.

- páncreas: 0,2 gr.

- suprarrenales: I,7 gr y I gr.

- riñón: 2,4 gr y 2 gr.

En vértex se localizó un foco de cefalohematoma y los órganos internos presentaban signos de inmadurez, sin malformaciones cardiacas aparentes. No existían signos de maceración visceral. La placenta, de 162 gr de peso, no presentaba más hallazgos macroscópicos que signos de infiltración hemática en los bordes placentarios.

Los estudios histopatológicos practicados posteriormente en el Instituto Nacional de Toxicología y Ciencias Forenses (INTCF) de Madrid arrojaron los siguientes hallazgos:

- Inmadurez histológica general

- Encéfalo con una amplia matriz germinal periventricular y con un foco de hemorragia.

- Pulmones en fase canalicular, sin infiltrados ni signos de aspiración de líquido amniótico 
- Hígado con intensa hematopoyesis y espacios porta presentes siendo solo reconocibles los de gran tamaño.

- Riñón con gruesa capa nefrogénica superficial y 6 filas de glomérulos ya desarrollados.

- Placenta: depósito de fibrina subcorial; vellosidades con estroma reticular con canales y células de Hofbauer y escaso desarrollo capilar. No había vellosidades terminales.

Los análisis biológicos practicados sobre sangre materna en relación con vellosidades coriónicas y muestra de esternón procedentes de la placenta y el feto indicaron una coincidencia entre estas muestras tras el estudio de STR de 15 alelos cromosómicos y el gen de amelogenina (maternidad prácticamente probada, según los predicados de Hummel [4], con una probabilidad de no exclusión del 99,989\% y un índice de maternidad de 9460: I).

Los estudios toxicológicos realizados sobre muestras de sangre y orina de la supuesta madre indicaban la existencia de restos de metadona, EDDP (metabolito de la metadona) y benzoilecgonina (metabolito de cocaína). En hígado fetal se identificaron benzoilecgonina (I,25 $\mu \mathrm{g} / \mathrm{g})$ y metadona $(0,46 \mu \mathrm{g} / \mathrm{g})$.

Se intentó practicar un estudio específico de restos de misoprotol en sangre y orina maternas, placenta y líquido intervisceral, riñón e hígado fetales. El INTCF no disponía de los reactivos necesarios que solo estaban disponibles a través de la casa comercial que fabrica el producto, laboratorios Searle. Finalmente, pudieron conseguirse los reactivos, si bien únicamente para el misoprotol pero no para sus metabolitos principales. Se intentó el análisis químico con un límite de detección de Misoprostol de $0.5 \mu \mathrm{g} / \mathrm{mlit}$, siendo los análisis negativos.

Las conclusiones iniciales de la investigación, a salvo de la revisión bibliográfica posterior, fueron:

- Feto fallecido por posible causa materna

- Data de la muerte en torno a 24 horas (no se encontraron referencias bibliográficas específicas sobre parámetros de evolución postmortem en el caso de fetos)

- Edad gestacional de 21 a 24 semanas (feto no viable)

- Datos indicativos de consumo materno de metadona y cocaína anteparto.

- Sospecha no confirmada de consumo de Misoprostol como agente inductor del parto.

\section{DISCUSIÓN:}

El Misoprostol [5] es un producto químico análogo de la Prostaglandina EI. Ya desde las primeras referencias bibliográficas publicadas en 1981 como SC-29333, el producto demostró su eficacia como agente protector gástrico especialmente en casos de ulcerogénesis inducida por AAS en ensayos en animales [6]. El primer ensayo en humanos fue publicado en 1982 [7].

El Vademecum Internacional en su versión 2003 seguía indicando que el producto estaba indicado únicamente como agente antiulceroso y como profiláctico de gastritis y ulcus gastroduodenal. La dosis diaria recomendada con estos fines es de $200 \mu \mathrm{g} 2$ a 4 veces al día. Los efectos adversos descritos son diarreas, náuseas, cefaleas, vértigos y dolor abdominal. Como advertencia marginal se indica que el producto "no se debe consumir durante el embarazo, por su acción sobre el músculo liso". Según se indica en el prospecto del producto comercial, este era un producto de venta exclusiva con receta médica. En 1998, el INTCF no disponía de datos indicativos en su centro de información toxicológica de otros datos distintos de los apuntados. 
En la versión actual del Vademecum [8] el Cytotec ${ }^{\circledR}$ ha sido eliminado de la lista de productos publicados, pero permanece como Misoprostol en la fórmulas de productos como Artrotec ${ }^{\circledR}$ (laboratorios Pharmacia Spain) y Normulen ${ }^{\circledR}$ (Laboratorios Grunenthal) en sus formas de comprimidos entéricos, indicando en sus contraindicaciones que "está contraindicado en las mujeres gestantes y en las que piensen quedar embarazadas, ya que puede aumentar el tono y contracciones uterinas lo que conduciría al aborto". En la base de datos del Consejo General de Colegios Oficiales de Farmacéuticos (BOT), el Misoprostol se especifica comercializado tanto en forma única, bajo las denominaciones de Glefos ${ }^{\circledR}$ (Laboratorios Grunenthal), Cytotec ${ }^{\circledR}$ (Laboratorios Pharmacia Spain) y Misoprostol ${ }^{\circledR}$ (genérico), como en forma de asociación en las dos especialidades ya indicadas, Artrotec ${ }^{\circledR}$ y Normulen ${ }^{\circledR}[9]$. En esta base de datos, solo en el caso del producto genérico se especifica que el producto se considera dentro de la categoría $X$ de la FDA por el riesgo de teratogenicidad [ 10$]$. El resto de presentaciones comerciales no hacen referencia a dicho riesgo teratógeno [9] y no se especifica el uso del producto como abortivo.

En USA, actualmente, la FDA (Food and Drug Administration) no reconoce otro uso autorizado de este producto que el ya indicado como protector gástrico. Sin embargo, el Misoprostol es distribuido comercialmente tanto en formas aisladas como en asociación con antinflamatorios.

La literatura médica empieza a destacar en el año 199| el uso indebido del Misoprostol como agente abortivo. En este año, la revista The Lancet en su número 337 [ I I ] ya publica un estudio sobre la posible relación entre el uso inadecuado del Misoprostol como abortivo y el desarrollo de malformaciones fetales. Múltiples trabajos, especialmente originarios de Brasil [12], indican que el uso de este producto fuera del contexto médico con fines abortivos es una realidad alarmante. En el siguiente número de The Lancet, un trabajo de revisión analiza ya los aspectos éticos y legales del uso de este producto con fines abortivos legales en el propio Reino Unido. El debate abierto y enconado sobre el uso de esta sustancia con tales fines está en las páginas de opinión de las principales revistas médicas anglosajonas desde entonces. Una simple búsqueda en internet en un buscador general permite localizar rápidamente páginas que aseguran la venta del Cytotec con fines abortivos y su envío a cualquier lugar del mundo sin mediación de receta médica alguna. No obstante, hasta la fecha, el laboratorio Pharmacia (Pfizer) responsable original de su comercialización solo ha hecho público que el producto está siendo usado con fines no recomendados por la casa comercial y lo ha notificado a todos los ginecólogos y obstetras, posiblemente como reacción a las presiones de los grupos antiaborto de USA [13].

En la actualidad, el Misoprostol es un agente utilizado de forma rutinaria por los servicios de obstetricia como agente inductor del parto y en la realización de interrupciones voluntarias del embarazo. Se han recomendado pautas en el medio sanitario para la provocación del aborto durante el primer trimestre de gestación de $400 \mu \mathrm{g}$ por vía vaginal y posterior aspiración de restos fetales [14]. Esta pauta es eficaz para la provocación de dilatación cervical y estimulación del músculo liso. Durante el segundo trimestre, la dosis recomendada ha sido de $800 \mu \mathrm{g}$ por vía oral, seguidos de dosis de $400 \mu \mathrm{g}$ cada 8 horas [15]. La eficacia como inductor del parto en todas las series es superior al $90 \%$, incluso en el tercer trimestre de gestación [16]. El tiempo medio entre la administración de la sustancia por vía oral o vaginal y la aparición del efecto de inducción de dilatación cervical es de media hora a 3 horas.

Sin embargo, son innumerables las referencias sobre complicaciones severas en el caso de un uso inadecuado de esta sustancia en el medio extrahospitalario (tabla I). Entre las complicaciones más destacadas para el feto se hayan riesgo de desarrollo de síndrome de Moebius (parálisis 
facial congénita) [17], anomalías embrionarias múltiples, equinovaro congénito con defectos de pares craneales, artrogriposis de extremidades inferiores, defectos de cadera, anomalías craneofaciales congénitas [18]. Algunos estudios sugieren que la dosis habitual recibida en estos casos pudo de ser de $800 \mu \mathrm{g}$ por vía oral y superior [19]. Entre las complicaciones para la madre, todas las series destacan que la principal complicación es el sangrado incoercible postparto, si bien en una proporción baja de casos y manejable con medios farmacológicos convencionales en el medio hospitalario. Existen algunas referencias que apuntan la posibilidad de relación entre el uso del Misoprostol como agente inductor del parto y casos de rotura uterina [19].

\begin{tabular}{|l|}
\hline Malformaciones fetales: \\
\hline Síndrome de Moebius \\
\hline Artrogriposis \\
\hline Equino-varo asociado con deficiencia de pares craneales (especialmente V, VI, VII) \\
\hline Hidrocefalia congénita \\
\hline Defectos de cadera \\
\hline Bandas de contracción en dedos de las manos \\
\hline Sindactilia \\
\hline Amioplasia en extremidades inferiores \\
\hline Onfalocele \\
\hline Complicaciones maternas: \\
\hline Sangrado uterino postparto incoercible \\
\hline Roturas uterinas intraparto \\
\hline
\end{tabular}

Tabla I. Complicaciones descritas por uso inadecuado de Misoprostol durante la gestación $[11,12,17,18,19]$

Existen pocos estudios sobre la toxicocinética del Misoprostol. La ficha técnica de Laboratorios Searle en 1999 (actualmente, laboratorios Pfizer, dentro del grupo Pharmacia) [20] indicaba que el Misoprostol es un fármaco de elevada biodisponibilidad; su pico máximo plasmático en pauta oral se alcanza en 12 +/- 3 minutos. El producto tras su absorción en forma ácida es metabolizado en el hígado [2 I]. La vida media de eliminación plasmática es de apenas 30 minutos y en las especies estudiadas (no en el ser humano) las dosis de $200 \mu \mathrm{g}$ por vía oral producen cifras despreciables bajo la forma de Misoprostol en plasma a los 30 minutos de su administración. La eliminación del producto es por vía urinaria en un 73 \% y bajo la forma de metabolitos. La mayoría de estos corresponden con metabolitos polares del ácido SC-30695, el principal metabolito de este análogo prostaglandínico.

En resumen, el Misoprostol desde el punto de vista médico es, además de una eficaz agente protector gástrico:

- Un inductor del parto eficaz (>90\%) en cualquier trimestre del embarazo.

- Con una Biodisponibilidad rápida (10 mins).

- Con un Metabolismo rápido (30 mins). 
- De eliminación renal como metabolitos.

- Accesible al público general.

- Difícil de detectar de rutina por el INTCF.

- Con severos efectos adversos fetales y maternos si no se usa en las dosis recomendadas.

En el caso que se presenta, además del posible uso de Misoprostol, podría apuntarse como posible causa materna de tipo tóxico en la precipitación del aborto el consumo concomitante de metadona y cocaína por parte de la madre durante la gestación.

Existen múltiples referencias bibliográficas que apuntan la posibilidad de correlación entre el consumo de estas sustancias por parte de madres gestantes y el desarrollo de malformaciones fetales e incluso partos prematuros (tabla 2). Se ha sugerido el posible efecto depresor sobre el corazón fetal de la combinación entre etanol y cocaína como causa de muerte fetal [22]. En los hijos de madres consumidoras de cocaína, se ha descrito el desarrollo de abruptio placentae [23] y anomalías del tracto genitourinario [24], precipitación de reacciones de inmunización Rh (hydrops fetalis) [25], defectos congénitos de cadera, atresia intestinal e infarto intestinal [26] y bajo peso al nacimiento o microcefalia [27], así como otras anomalías con baja evidencia de correlación como defectos de cierre de tubo neural, SIDS, infartos cerebrales perinatales [24]. El consumo de cocaína durante la gestación se ha asociado, también con partos prematuros [28], abortos espontáneos por disminución de flujo uterino [29,30] y muertes fetales intraútero [3I]. En hijos de madres consumidoras de metadona se ha indicado la posibilidad de embarazo ectópico, abortos espontáneos o partos pretérmino [32].

\begin{tabular}{|l|}
\hline Abortos espontáneos por disminución de flujo uterino \\
\hline Muerte fetal intraútero \\
\hline Síndrome de muerte súbita del lactante \\
\hline Partos prematuros \\
\hline Retardo de crecimiento fetal intrauterino \\
\hline Abruptio placentae \\
\hline Reacciones de inmunización Rh (hydrops fetalis) \\
\hline Malformaciones del aparato génito-urinario \\
\hline Malformaciones esqueléticas \\
\hline Malformaciones del SNC \\
\hline Atresia intestinal y anal \\
\hline
\end{tabular}

Tabla II. Complicaciones descritas en casos de exposición a cocaína durante la gestación. [22-32].

Sin embargo, existen también múltiples referencias que ponen en duda la existencia de una clara correlación entre estos fenómenos. Los estudios de metanálisis y algunos estudios retrospectivos realizados sobre la posible correlación entre el consumo de metadona y cocaína y el desarrollo de malformaciones fetales o partos pretérmino no han podido concluir que existan evidencias claras de tal correlación [33, 34]. Los posibles casos descritos de correlación observada son explicados en estos metanálisis como errores de diseño de los anteriores estudios, al existir una posible confusión en estas series que no consideran que el perfil de las madres consumidoras corresponde, además, con el de madres que suelen provenir de un bajo estrato social, con un bajo control sani- 
tario general y obstétrico, bajo control pediátrico, consumo de otras sustancias asociadas y habitualmente afectas de infecciones por virus VHB, VHC, VIH y ETS (Sifflis).

Por tanto, en relación con el consumo de estas sustancias, puede concluirse que:

- En madres consumidoras habituales de cocaína y metadona no existen evidencias absolutas de correlación con abortos espontáneos y SIDS.

- Existe una evidencia discutida de correlación con anomalías congénitas fetales.

- No existen referencias de utilización de estas sustancias como inductoras de parto prematuro.

Desde el punto de vista médico forense, el posible origen tóxico del aborto debe intentar resolverse siguiendo unos parámetros de interpretación de los hallazgos patológicos y toxicológicos basado en un criterio científico aceptable. En este sentido, pueden resultar aceptables los criterios de causalidad en los casos de muertes tóxicas propuestos por Irey [35] (Criterio temporal, Criterio de latencia, Criterio de exclusión, Criterio de detención y reinicio, Criterio de patrón, Criterio de cuantificación, Criterio de identificación). Estos criterios permiten valorar el nivel de probabilidad de causalidad de una muerte tóxica según varios niveles de evidencia: Nivel de causalidad, Nivel de probabilidad, Nivel de posibilidad, Nivel de coincidencia y Nivel de negación. Una metodología similar de interpretación de resultados fue utilizada por los autores en un estudio previo [36].

En el caso que nos ocupa, se da la circunstancia de que sobre la base de los datos disponibles, en el caso de carecer de una declaración positiva de la madre refiriendo consumo previo de la sustancia, el nivel de probabilidad estimado sería de negación. Por otra parte, el nivel de evidencia en el caso de la hipótesis de un posible origen tóxico asociado con el consumo de metadona y cocaína sería de posibilidad. Del mismo modo, la propia hipótesis de un posible aborto espontáneo se hallaría en el mismo nivel de probabilidad de simple posibilidad [37].

En estas condiciones, dado que la madre en su comparecencia ante el juez y asistida por su letrado negó el consumo referido a los agentes, la posibilidad de valoración técnica de una posible conducta valorable como ilegal según el código penal vigente resulta dudosa. Sin embargo, el posible uso como abortivo de una sustancia de venta autorizada en España con fines diversos resulta éticamente complejo.

La alta fiabilidad del producto con fines inductores del parto en los tres trimestres de la gestación y su alta seguridad con las pautas recomendadas en el entorno hospitalario y obstétrico, convierten al Misoprostol en una abortivo legal ideal, a la par de mucho más barato que los medios oficialmente disponibles [13]. Sin embargo, su rapidez de acción y de metabolización y la limitación técnica de los laboratorios de referencia para su identificación rutinaria o extraordinaria, lo convierten en un abortivo ilegal ideal. Además, su virtual disponibilidad libre en el mercado vía internet o en el caso de receta médica con otros fines lo hacen fácilmente accesible para el público en general sin la adecuada supervisión médica. De la misma manera, su comercialización en España en combinación con otros fármacos con dosis recomendadas cercanas a los efectos abortivos y teratogénicos supone un riesgo adicional de desarrollo de abortos inducidos de forma accidental y de desarrollo de malformaciones fetales.

Es precisamente este último el mayor dilema ético, dado que las evidencias parecen claras en relacionar la administración inapropiada de este producto en dosis inadecuadas y sin una correcta supervisión médica con la aparición de severas malformaciones y anomalías fetales y posibles riesgos para la madre [38]. Resulta sorprendente que en las mismas fechas de los hechos que iniciaron 
esta investigación, existiese un enconado debate público sobre la conveniencia de comercializar en nuestro país la famosa RU-486 (mifepristone) y, al tiempo, en nuestro mercado ya se estuviese disponiendo de una sustancia al menos tan eficaz como la debatida, más barata y que de hecho ya se estaba utilizando con fines abortivos tanto en nuestro país como en países extranjeros fuera del control médico estricto del que una sustancia de estas características debiera disponer. Incluso, en el año 1997, una sentencia del Tribunal Superior de Justicia de la Comunidad Valenciana indicaba que el uso de este producto con fines abortivos no autorizados oficialmente ni recomendados por el laboratorio productor podría ser considerado legal dados "sus resultados buenos y positivos para la salud de las mujeres" [39]. Y esta situación, a día de hoy y aún metamorfoseada en asociación farmacológica [8], persiste en múltiples países, el nuestro entre ellos [40].

Parafraseando al Dr. MacKenzie [13], el asunto del Misoprostol nos plantea un curioso dilema. Cuando una droga demuestra ser equivalente a otras drogas en una cierta acción terapéutica, con una aceptable margen de seguridad en manos adecuadas, pero resulta cien veces más barata que aquellas, sería lógico utilizar la droga más barata. Sin embargo, puede que el laboratorio que la produce y las autoridades no apoyen su uso con estos fines, sino con unos diversos, pese al riesgo contrastado para las mujeres gestantes y su descendencia. Incluso, puede que para las indicaciones autorizadas existan productos más eficaces y con mayor margen de seguridad. Pero, además, puede que esta sustancia resulte difícilmente detectable en laboratorios toxicológicos y, por ello, su posible uso no autorizado resultaría difícilmente demostrable para la Justicia. Este es el caso del Misoprostol.

La pregunta que queda por contestar en este dilema es quién puede salir beneficiado de una situación como esta, en la que tanto los pacientes, como sus descendientes y las propias autoridades sanitarias y judiciales resultan claramente perjudicados.

La necesidad de una adecuada recalificación de este producto farmacológico y su control médico estricto resulta aún más evidente en países del entorno africano, asiático y latinoamericano, donde la tasa de abortos sin control sanitario es muy elevada y la mortalidad materna y morbilidad perinatal son excesivas. En estos países, esta recalificación resultaría en una evidente mejora de la salud pública con un coste social mínimo frente a otros métodos abortivos [4I].

\section{BIBLIOGRAFÍA:}

I. Grimes DA. Unsafe abortion: the silent scourge. Br Med Bull 2003; 67:99-II3.

2. Coelho HLL, Misago C, Fonseca WVC, Souza DSC, Araujo JML. Selling abortifacients over the counter in pharmacies in Fortaleza. Lancet 1991; 338: 247.

3. Medicina Legal y toxicología. Gisbert Calabuig. 6 a edición. Editor: Villanueva E. Ed. Masson 2004.

4. Huguet F. Parámetros estadísticos en genética forense. Investigación de la paternidad. En: Martínez Jarreta MB. La prueba de ADN en medicina forense. Masson. 1999.

5. Ficha técnica del Cytotec ${ }^{\circledR}$ de laboratorios Pfizer (anteriormente laboratorios Searle).

http://www.pfizer.com/download/uspi_cytotec.pdf

6. Larsen KR, Jensen NF, Davis EK, Jensen JC, Moody FG .The cytoprotective effects of (+/-)-15-deoxy-16-alpha, beta-hydroxi-16methyl PGI methyl ester (SC-29333) versus aspirin-shock gastric ulcerogenesis in the dog. Prostaglandins 1981;2I(suppl):I19-24.

7. Akdamar K, Agrawal N, Ertan A. Inhibition of nocturnal gastric secretion in normal human volunteers by Misoprostol, a synthetic prostagalndin El methyl ester analog. Am J Gastroenterol 1982;77(I2):902-4.

8. Vademecum internacional. Versión española online. http://vademecum.medicom.es/

9. Base de Datos del Consejo de Colegios Oficiales de Farmacéuticos de España (BOT). http://www.portalfarma.com/

10. Clasificación de los medicamentos teratogénicos según la FDA. Boletín Oficial de Farmacovigilancia. 1998,16. http://www.cfnavarra.es/bif/default.htm

II. Schonhofer PS. Misuse of Misoprostol as an abortifacient may induce malformations. Lancet 1991;337:1534.

12. Fonseca WVC, Alencar AJC, Mota FSB, Coelho HLL. Misoprostol and congenital malformations. Lancet 1991;338:56.

13. Mackenzie WE. Misoprostol and the politics of fear. Lancet 2001;357(9264):1296. 
14. Singh K, Fong YF, Prasad RN, Dong F. Randomized trial to determine optimal dose of vaginal Misoprostol for preabortion cervical priming. Obstet Gynecol 1998; 92(5):795-8.

15. Feldman DM, Borgida AF, Rodis JF, Leo MV, Campbell WA. A randomized comparison of two regimens of Misoprostol for second-trimester pregnancy termination. Am J Obstet Gynecol 2003 Sep;189(3):710-3.

16. Sánchez.-Ramos L, Hsieh E. Pharmacologic methods for cervical ripening and labor indiction. Curr Womens Health Rep 2003;3:55-60. 17. Sanchez 0, Guerra D. Síndrome de Moebius: fetopatía por Misoprostol. Reporte de un paciente. Investi Clin 2003; 44(2). http://www.scielo.org/index.php?lang =es

18. Gonzalez CH, Marques-Dias MJ, Chong Ae kim, Sugayama SMM, Da Paz JA, Huson SM, Holmes LB. Congenital abnormalities in Brazilian children associated with Misoprostol misuse in first trimester of prgnancy. Lancet 1998;351:1624-7.

19. Plaut M, Schwartz M, Lubarsky S. Uterine rupture associated with the use of Misoprostol in the gravid patient with a previous cesarean section. Am J Obstet Gynecol 1999; 180: 1535-40.

20. Ficha técnica del Cytotec ${ }^{\circledR}$ remitida por laboratorios Searle el 14-6-1999 a los juzgados de Bilbao.

21. Goldberg AB, Carusi DA, Meckstroth. Misoprostol in Ginecology. Curr Womens Health Rep 2003;3:475-483.

22. Richards IS. Ethanol potentiates the depressant effects of cocaine in human fetal myocardium in vitro. J Toxicol Clin Toxicol 1997;35(4):365-9.

23. Acker D, Sachs BP, Tracey KJ, Wise WE. Abrutio placentae associated with cocaine use. Am J Obstet Gynecol 1983; 146(2):220-22I.

24. Slutsker $L$. Risks associated with cocaine use during pregnancy. Obstet Gynecol 1992;79(5):778-89.

25. Bowman J, Harman C, Manning F, Menticoglou S, Pollock J. Intravenous drug abuse causes Rh inmunization. Vox Sang |99|;61(2):96-8.

26. Hoyme HE, Jones KL, Dixon SD, Jewett T, Hanson JW, Robinson LK. Prenatal cocaine exposure and fetal vascular disruption. Pediatrics 1990;85(5):743-7.

27. Hadeed AJ, Siegel SR. Maternal cocaine use during pregnancy: effect on the newborn infant. Pediatrics 1989;84(2):205-10.

28. Das G. Cocaine abuse and reproduction. Int J Clin Pharmacol Ther 1994; 32(I):7-II.
29. Mittleman RE, Cofino JC, Hearn WL. Tissue distribution of cocaine in a pregnant woman. J Forensic Sci 1989:48I-6.

30. Nolte KB. Cocaine, fetal loss and the role of the forensic pathologist. J Forensic Sci 1991; 36(3): 926-9.

3I. Morild I, Stajic M. Cocaine and fetal death. For Sci Int 1990; 47:18I-189.

32. Selwyn PA, Schoenbaum EE, Davenny K, Robertson V], Feingold AR, Shulman JF, Mayers MM, Klein RS, Friedland GH, Rogers MF. Prospective study of human inmunodeficiency virus infection and pregnancy outcomes in intravenous drug users. JAMA 1989;261(9):1289-94.

33. Lutiger B, Graham K, Einarson TR, Koren G. Relationship between gestational cocaine use and pregnancy outcome: a metaanalysis. Teratology 1991;44(4):405-14.

34. Cartwright PS, Schorge J0, McLaughlin FJ. Epidemiologic characetristics of drug use during pregnancy: experience in a Nashville hospital. South Med J 1991;84(7):867-70.

35. Irey NS: When is a disease drug induced? En: Riddell RH. Pathology of drug-induced and toxic diseases. Churchill Livingstone, 1982. pp I-I8.

36. Garamendi PM, Sánchez de León MS. Mortalidad asociada con la contaminación atmosférica por S02. A propósito de un caso de autopsia médico legal tras un episodio de polución atmosférica. Cuad Med Forense 2003; 33: 43-56.

http://www.scielo.org/index.php?lang =es

37. Ovalle A, Kakarieka E, Vial MT, González R, Correa A, Sukni M, Figueroa J. Histopatología del aborto espontáneo entre 12 y 22 semanas. Rev Chil Obstet Ginecol 2003;68(5):361-370. http://www.scielo.org/index.php?lang = es

38. Wagner M. Misoprostol and the politics of convenience. Lancet 2001;357(9274).

39. Carbonell i Esteve JL. Interrupción voluntaria del embarazo ¿Aborto farmacológico?. V Congreso de la Sociedad Española de Contracepción. http://www.sec.es/p_ponen.htm

40. Sindicato médico del Uruguay. Resúmenes de prensa (Navarra G. "Usar un analgésico para provocar abortos". La Nación. 19-I2004) http://www.smu.org.uy/titulares/enlaces.php?id $=23826$

4I. Florence M, Weeks A, Fiala C, Safar P. Misoprostol is an essential drug. Misoprostol in Obstetrics and Gynaecology. http://www.misoprotol.org/ 